\title{
REVIEW
}

\section{Molecular aspects of type 1 diabetes}

M A Kelly, M L Rayner, C H Mijovic, A H Barnett

J Clin Pathol: Mol Pathol 2003;56:1-10

Type 1 diabetes is a T cell mediated autoimmune disease, characterised by the selective destruction of pancreatic $\beta$ cells, and susceptibility is determined by a combination of genetic and environmental factors. The environmental agents implicated include viruses and dietary factors, although none has yet been shown to be directly responsible for triggering $\beta$ cell autoimmunity. The genetic factors that influence disease risk have been subjected to more intensive study and two gene regions of major importance have been identified: the human leucocyte antigen locus and the insulin gene. This review will focus on the mechanisms by which these genes might influence the risk of developing type 1 diabetes.

See end of article for authors' affiliations

......................

Correspondence to: Dr M A Kelly, Department of Medicine, Clinical Research Block, Queen Elizabeth Hospital, Edgbaston, Birmingham B15 2TH, UK; m.a.kelly@bham.ac.uk

Accepted for publication 2 October 2002
$\mathrm{T}$ ype 1 diabetes is a multifactorial autoimmune disease, which is characterised by $\mathrm{T}$ cell mediated destruction of the insulin secreting $\beta$ cells of the islets of Langerhans in the pancreas. The destructive process leads to severe insulin depletion, which results in hyperglycaemia, because of hepatic overproduction of glucose by glycogenolysis and gluconeogenesis and decreased cellular uptake of glucose from the circulation. In the absence of insulin, there is also an increase in fat breakdown and fatty acid oxidation, resulting in the excessive production of ketones. If left untreated, these metabolic disturbances lead progressively to central nervous system depression, coma, and death. Therefore, the disease requires life long treatment with exogenous insulin for survival. The rate of $\beta$ cell destruction varies from patient to patient, but tends to be more aggressive in infants and young children. ${ }^{1}$ Hence, type 1 diabetes usually presents during childhood or adolescence, although it may develop much later in life. The variation in age at onset could be indicative of disease heterogeneity, with different mechanisms leading to $\beta$ cell destruction in childhood onset versus adult onset diabetes. This might reflect the involvement of different genetic and/or environmental susceptibility determinants.

\section{"Animal studies have also shown that $T$ cells play an important role in the disease pathogenesis"}

The early stages of the disease process leading to type 1 diabetes are characterised by insulitis, the infiltration of the pancreatic islets by mononuclear immune cells, including dendritic cells, macrophages, and T cells. ${ }^{23}$ Although this could reflect a normal inflammatory response to tissue damage, perhaps induced by exogenous factors such as viral infections, the lymphocytic infiltrate is thought to contribute directly to $\beta$ cell destruction. In support of this hypothesis, autoreactive $\mathrm{T}$ cells specific for $\beta$ cell proteins (including insulin, glutamic acid decarboxylase (GAD), and the protein tyrosine phosphatase, IA-2) have been isolated from the peripheral blood of newly diagnosed individuals with diabetes. ${ }^{4-8}$ Some of these T cells have been shown to be capable of destroying $\beta$ cells in vitro.' Animal studies have also shown that $\mathrm{T}$ cells play an important role in the disease pathogenesis. The non-obese diabetic (NOD) mouse spontaneously develops insulin deficient diabetes that shares many immunological and pathological features with type 1 diabetes. ${ }^{10}$ The development of disease in this animal has been shown to be thymus dependent and to require both $\mathrm{CD} 4$ positive and CD8 positive $\mathrm{T}$ cells. Furthermore, some of the autoreactive $\mathrm{T}$ cells isolated from diabetic NOD mice are capable of transferring the disease to non-diabetic animals and accelerating the onset of diabetes in NOD neonates. ${ }^{11-13}$

The autoimmune aetiology of type 1 diabetes is also reflected by the presence of circulating autoantibodies, specific for $\beta$ cell proteins including insulin, GAD, and IA-2. These autoantibodies are detectable in $85-90 \%$ of subjects with diabetes at the time of diagnosis. ${ }^{14}{ }^{15}$ It is unclear whether they participate directly in $\beta$ cell destruction or arise secondary to the release of autoantigens from islets damaged by other components of the immune system. They are, however, good markers of the underlying disease pathogenesis. The appearance of the autoantibodies precedes the clinical onset of disease, often by several years. Indeed, they can develop as early as the 1st year of life, with most individuals possessing autoantibodies directed towards multiple $\beta$ cell targets by the time clinical symptoms become apparent. ${ }^{16}$ Therefore, the presence of multiple autoantibodies can be used as a sensitive marker to predict the risk of developing type 1 diabetes, ${ }^{17}{ }^{18}$ although there are some autoantibody positive individuals who do not progress to the disease. Such individuals are particularly useful for the study of factors that protect against the development of diabetes.

Abbreviations: GAD, glutamic acid decarboxylase; HLA, human leucocyte antigen; IDDM, insulin dependent diabetes mellitus; IGF, insulin-like growth factor; $M H C$ major histocompatibility complex; NOD, non-obese diabetic; TCR, T cell receptor; VNTR, variable number of tandem repeats 


\section{SUSCEPTIBILITY DETERMINANTS OF TYPE 1 DIABETES}

Susceptibility to type 1 diabetes is influenced by both genetic and environmental factors. The importance of inherited risk determinants is demonstrated by the clustering of the disease within families. The life time risk of diabetes among first degree relatives of diabetic individuals is $5-6 \%$, compared with approximately $0.4 \%$ in the general white population. ${ }^{19}$ Furthermore, the concordance rate for the disease is much higher among monozygotic twins (30-40\%) than dizygotic twins $(6 \%) .^{20}{ }^{21}$ Although this observation is indicative of a large genetic contribution to disease risk, the relatively low concordance rate among identical twins suggests that the susceptibility genes have low penetrance; that is, not all individuals who are genetically "at risk" of type 1 diabetes will develop the disease. Discordance between identical twins may reflect the generation of disparate immunological repertoires, through random rearrangement of the genes encoding $\mathrm{T}$ cell receptors and B cell receptors, stochastic events, or somatic mutations. Alternatively, it may indicate an important non-genetic (environmental) input to disease susceptibility. The importance of environmental determinants of disease risk is further supported by the seasonal variation in the incidence of diabetes, with most new cases occurring in autumn and winter, ${ }^{22}$ and the geographical variation in disease incidence. For example, the incidence of type 1 diabetes among French and Jewish children living in Canada has been reported to be higher than among their counterparts living in France or Israel. ${ }^{23}$ Overall, environmental factors are thought to account for up to two thirds of disease susceptibility.

\section{Environmental factors}

Several environmental agents have been suggested to contribute to the risk of developing type 1 diabetes. These include viral infections, dietary factors in early infancy, vaccination, climatic influences, toxins (for example, nitrosamines), and stress. $^{24-27}$ It is generally believed that the environmental agents trigger disease development in genetically susceptible individuals. However, recent observations suggest a more complex model in which exposure to multiple environmental factors throughout life influences the penetrance and expression of genetically determined immune dysregulation. This model is supported by the observation that multiple infections during the lst few years of life are associated with a decreased risk of developing type 1 diabetes, whereas an increased risk is associated with perinatal infections. ${ }^{27-29}$ This suggests that environmental factors may modify the developing immune system in an age dependent manner and may therefore promote or attenuate disease at different stages of development, depending upon the timing and number of exposures.

\section{"Infection with rubella virus or coxsackie B4 virus has been frequently associated with an increased risk of developing the disease"}

It is possible that type 1 diabetes has a heterogeneous aetiology, with different environmental factors promoting $\beta$ cell destruction via different mechanisms. Furthermore, these disparate environmental factors may interact with different genetic determinants to influence the overall risk of developing disease. This potential heterogeneity hampers the identification of disease susceptibility determinants.

Therefore, despite much research no environmental agent responsible for triggering type 1 diabetes has been conclusively identified, although infection with rubella virus or coxsackie B4 virus has been frequently associated with an increased risk of developing the disease. Indeed, circulating $\mathrm{T}$ cells specific for these viruses are more prevalent among patients with type 1 diabetes than healthy subjects. ${ }^{30} 31$
However, the mechanism by which the viruses lead to $\beta$ cell destruction is yet to be determined.

\section{Genetic factors}

The genetic determinants of susceptibility to type 1 diabetes are better understood than the environmental risk factors. The first diabetes susceptibility genes to be identified were the human leucocyte antigen (HLA) genes, located on chromosome $6 \mathrm{p} 21 .{ }^{32-34}$ Subsequent studies demonstrated an association between the disease and the insulin gene region on chromosome 11 p. $^{35}{ }^{36}$ In the mid to late 1990s, high throughput screening of the entire human genome in families with two or more affected siblings was used to identify additional chromosomal regions that may contain susceptibility genes for type 1 diabetes. ${ }^{37-40}$ Over 20 loci showed evidence for linkage with the disease in different data sets. All the studies consistently reported linkage to the HLA gene region (designated IDDM1). Several genome screens, in combination with family based association studies, also supported a role for the insulin gene region (designated IDDM2) in disease susceptibility. ${ }^{37}{ }^{31}$ Linkage to eight additional loci was replicated in independent data sets: IDDM4 (chromosome llq13), IDDM5 (chromosome 6q25), IDDM7 (chromosome 2q31), IDDM8 (chromosome 6q27), IDDM10 (chromosome 10p1 1-q11), IDDM12 (chromosome 2q33), IDDM13 (chromosome 2q35), and IDDM15 (chromosome 6q21). ${ }^{37-40} 4243$ In addition, the locus designated IDDM6 (chromosome 18q21) showed consistent evidence for association with the disease in family studies. ${ }^{44}$ Although the chromosomal locations of these loci are known, the precise identity of the susceptibility genes in these regions remains to be determined.

The genome screens confirmed that the IDDMI locus (the HLA gene region) is the major genetic determinant of disease risk, accounting for $42 \%$ of the familial inheritance of type 1 diabetes. $^{37}$ The IDDM2 locus (the insulin gene region) contributes a further $10 \%$ of genetic susceptibility. ${ }^{37}$ The remainder of this review article will focus on the mechanisms by which these genes might influence the risk of developing type 1 diabetes.

\section{IDDM1 (HLA) GENES AND TYPE 1 DIABETES}

The IDDMI susceptibility locus encompasses the HLA genes, located within the major histocompatibility complex (MHC). The MHC spans a 3.5 megabase region of chromosome 6p21 and consists of over 200 genes arranged into three subregions, class I, class II, and class III (fig 1).

(1) The class I genes encode $\alpha$ peptide chains, which associate with $\beta_{2}$ microglobulin to form the class I molecules (fig $2 \mathrm{~A}$ ). These are expressed on the surface of all nucleated cells and play a crucial role in the restriction of cytotoxic $\mathrm{T}$ cell activity. The HLA class I molecules bind to peptide fragments derived from endogenous antigens and present them for recognition by the T cell receptors (TCRs) of CD8 positive T cells.

(2) The class II (HLA-D) loci are subdivided into at least one A and one $\mathrm{B}$ gene. These encode the $\alpha$ and $\beta$ peptide chains, respectively, which combine to form the heterodimeric class II molecules (fig 2B). The expression of these molecules is normally restricted to professional antigen presenting cells, B cells, and activated T cells. The HLA-DR, HLA-DQ, and HLA-DP molecules are involved in the activation of helper $\mathrm{T}$ cells. The $\alpha \mathrm{l}$ and $\beta \mathrm{l}$ domains of these molecules form a cleft into which peptide fragments derived from exogenous antigens can bind. These peptides are then presented for recognition by the TCRs of CD4 positive T cells (fig 3). The recognition process activates the responding $\mathrm{T}$ cells and initiates an immune response.

(3) The class III genes encode a range of molecules with a variety of functions, including complement components ( $\mathrm{C} 2$, $\mathrm{C} 4$, and $\mathrm{Bf}$ ), tumour necrosis factor, and heat shock protein, Hsp70. 


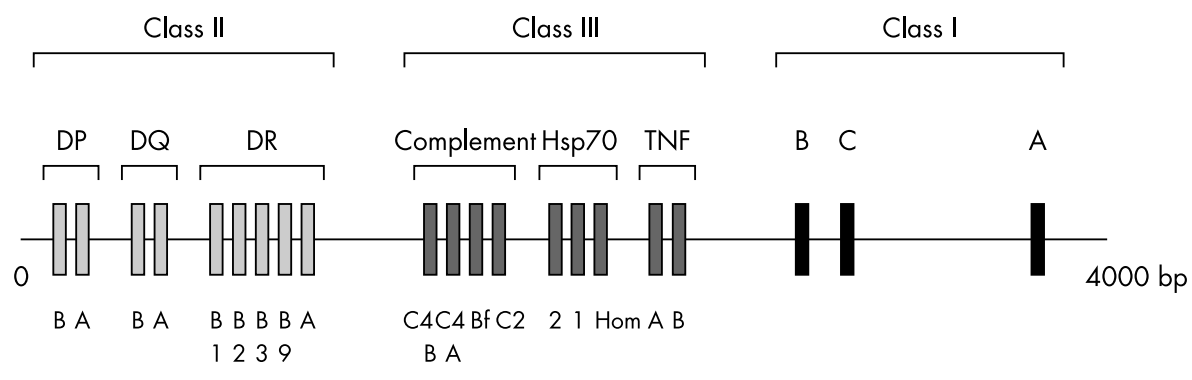

Figure 1 A simplified map of the major histocompatibility complex on chromosome 6p21, showing the genes arranged in three classes. Hsp, heat shock protein; TNF, tumour necrosis factor.

A

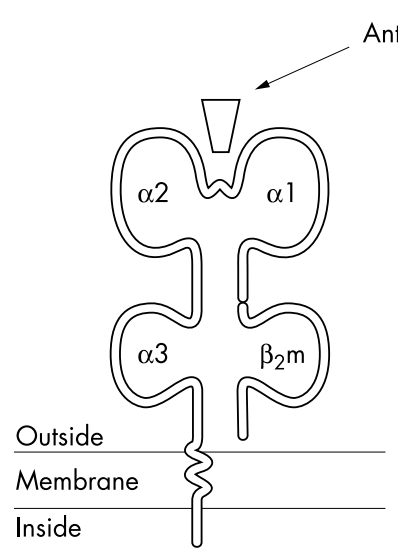

B

Figure 2 A diagrammatic representation of $(A)$ a human leucocyte antigen (HLA) class I molecule and (B) an HLA class II molecule, showing the antigen binding clefts.

Many of the HLA genes are highly polymorphic. The class I and class II molecules encoded by these allelic variants show great variability in their three dimensional structure, particularly in the antigen binding regions of the molecules. This has important functional consequences, because the structure of the antigen binding site determines the way in which the molecule interacts with a particular antigenic peptide and $\mathrm{T}$ cell receptor.
"The identification of the primary disease susceptibility determinants within the major histocompatibility complex region is confounded by strong linkage disequilibrium between the genes"

Because the HLA class I and class II molecules play a pivotal role in the activation of $\mathrm{T}$ cell responses, the genes encoding these molecules have been implicated in susceptibility to several $\mathrm{T}$ cell mediated autoimmune diseases, including type 1 diabetes. However, the identification of the primary disease susceptibility determinants within the MHC region is confounded by strong linkage disequilibrium between the genes-that is, particular alleles at distinct loci are inherited together more frequently than expected by chance. The combination of alleles inherited together on the same chromosome is known as a haplotype. It is difficult to distinguish the primary associations with disease susceptibility from associations occurring secondary to linkage disequilibrium. Despite this complication, a recent fine mapping study of the UK population suggested that the HLA class II genes, DRBI and DQBl, are the major determinants of IDDMl encoded susceptibility to type 1 diabetes. ${ }^{45}$ However, the disease risk conferred by these genes may be modified by other MHC loci, including the class I HLA-B gene and the class II HLA-DPBl gene. ${ }^{46} 47$

Susceptibility to type 1 diabetes is associated with two combinations of DQAl and DQBl alleles, namely: DQA ${ }^{*} 0501$.DQB $1 * 0201$ and DQA ${ }^{*} 0301$.DQB ${ }^{*} 0302$, which encode the HLA-DQ2 and HLA-DQ8 molecules, respectively. Two DRB1 alleles, $\mathrm{DRBI}^{*} 03$ and $\mathrm{DRB1}{ }^{*} 04$ (which encode the DR3 and DR4 molecules, respectively), are also associated with

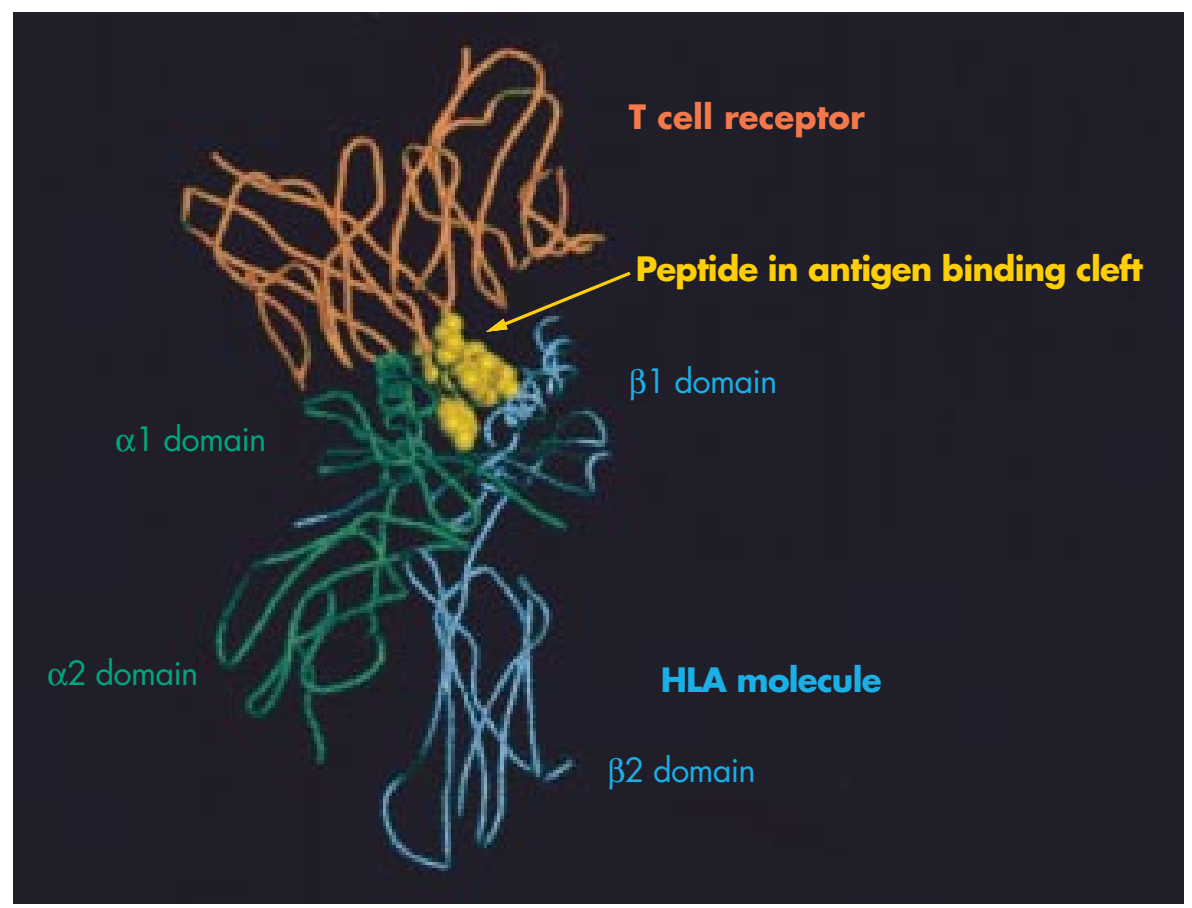

Figure 3 Antigen presentation to the T cell receptor of a CD4 positive helper $T$ cell by a human leucocyte antigen class II molecule. 
an increased risk of disease. $\mathrm{DRB} 1{ }^{*} 03$ is in linkage disequilibrium with the $\mathrm{DQAl}^{*} 0501$.DQB ${ }^{*} 0201$ allelic combination (forming the DR3.DQ2 haplotype), whereas $\mathrm{DRBl}^{*} 04$ is in linkage disequilibrium with DQA1*0301.DQB1*0302 (forming the DR4.DQ8 haplotype). Up to $90 \%$ of patients with diabetes carry one or both of these haplotypes and the highest genetic risk of the disease is conferred by the DR3.DQ2/ DR4.DQ8 heterozygous genotype. Although the DQ locus has been suggested to be more strongly associated with the disease than the DRBl gene, ${ }^{48}$ it is clear from recent studies that both loci are important for determining overall disease risk. This is illustrated by the DR4.DQ8 haplotype; although DQ8 is the principal disease determinant on this haplotype, its influence on disease risk may be modified by the DRBl subtype present. ${ }^{49}$ The $\mathrm{DRBI}^{*} 0401$, DRB1*0402, and DRB ${ }^{*} 0405$ subtypes have been reported to increase the risk of diabetes independent of DQ8, whereas DRBI*0403 and DRB ${ }^{*} 0406$ confer protection from the disease. ${ }^{4851}$ The protective effect of $\mathrm{DRB}{ }^{*} 0403$ can override the susceptibility conferred by DQ8, even in subjects carrying the high risk DR3.DQ2/DR4.DQ8 genotype..$^{53}$

Strong natural protection against type 1 diabetes is also conferred by the DQAl*0102.DQB1*0602 haplotype, which encodes the HLA-DQ6.2 molecule. This molecule occurs in approximately $20 \%$ of the healthy white population, but is rarely found among patients with diabetes. ${ }^{54}$ The protection provided by DQ6.2 appears to be dominant over the susceptibility conferred by other HLA markers, because individuals heterozygous for DQA $1 * 0102 . \mathrm{DQB} 1{ }^{*} 0602$ and a high risk HLA haplotype, such as DR3.DQ2 or DR4.DQ8, do not usually develop diabetes. ${ }^{55}$ The DQ6.2 molecule can prevent progression to overt diabetes even after the onset of islet autoimmunity, suggesting that it may have an immunomodulatory role. ${ }^{56}$ However, its influence is not absolute because patients with diabetes who are positive for DQ6.2 have been reported..$^{57} 58$

Although the DR3.DQ2 and DR4.DQ8 haplotypes are the major genetic determinants of disease risk, approximately $10 \%$ of white patients with diabetes carry neither of these markers. This figure is higher in non-white populations (up to $30 \%)$. Among these individuals, susceptibility to diabetes is conferred by other HLA haplotypes, including DRB1*0801.DQAl*0401.DQB1*0402 (DR8.DQ4), DRB1*0101. DQAl*0101. DQB ${ }^{*} 0501$ (DR1.DQ5), and DRB1*0901. DQA1*0301.DQB 1*0303 (DR9.DQ9). ${ }^{59-61}$ It is unclear whether type 1 diabetes in individuals lacking the DR3.DQ2 and DR4.DQ8 haplotypes results from the same mechanism as that in patients with one or both of the high risk markers, although the clinical profile of the disease is the same in both groups. It is possible that the different HLA associations reflect an interaction with different environmental triggers of the disease.

In conclusion, the HLA associations with type 1 diabetes are complex, with many haplotypes influencing disease risk. These form a hierarchy ranging from strongly protective (DQ6.2) to highly predisposing (DR3.DQ2/DR4.DQ8 heterozygosity).

\section{Functional evidence for the role of HLA-DR and HLA-DQ molecules in disease pathogenesis}

A direct role for HLA-DR and HLA-DQ molecules in the pathogenesis of type 1 diabetes was recently demonstrated in studies of HLA transgenic mice. The expression of human DR3 $\left(\mathrm{DRB}{ }^{*} 03\right)$ and/or DQ8 (DQAl*0301.DQB $\left.{ }^{*} 0302\right)$ in the B10 strain of mice was shown to induce a loss of immune tolerance to $\mathrm{GAD}$, a potential $\beta$ cell autoantigen. ${ }^{62}{ }^{63}$ Mice expressing both HLA molecules also developed spontaneous insulitis, although they did not progress to overt diabetes. ${ }^{62}$ No immune reactivity to GAD was seen in transgenic mice expressing DR3 or DQ8 in combination with DQ6.2 (DQAl*0102.DQB1*0602), however. ${ }^{63}$ This finding is consistent with the dominant protective role reported previously for DQ6.2. In a separate study of C57BL/6 HLA transgenic mice, the expression of DQ8 or DR4 (DRB1*0401) alone was shown to induce spontaneous autoimmune diabetes, but only in mice expressing the $\mathrm{T}$ cell costimulatory molecule, B7.1, on their pancreatic $\beta$ cells ${ }^{64}$

Although these studies support a direct role for the DR and DQ molecules in disease pathogenesis, they have not helped us to understand how the molecules influence disease risk. Furthermore, the findings of the different studies are not directly comparable because of the different methodological approaches taken. Therefore, further investigations are required to gain an insight into the mechanism by which the HLA molecules influence the development of diabetes.

\section{The effect of molecular structure on the function of HLA-DR and HLA-DQ}

The influence of the HLA-DR and HLA-DQ molecules on the risk of type 1 diabetes is probably related to their central role in antigen presentation and the activation of a helper $\mathrm{T}$ cell mediated immune response. This function is largely determined by the precise three dimensional structure of the antigen binding clefts of the molecules, formed by the $\alpha 1$ and $\beta 1$ domains of the peptide chains. This dictates the way in which each molecule interacts with antigenic peptides and the TCRs of responding $\mathrm{T}$ cells.

"The influence of the HLA-DR and HLA-DQ molecules on the risk of type 1 diabetes is probably related to their central role in antigen presentation and the activation of a helper T cell mediated immune response"

Studies using $x$ ray crystallography and computer modelling have suggested that HLA molecules associated with susceptibility to type 1 diabetes share similar chemical and geometric properties in their antigen binding clefts. These characteristics are strikingly different from those of protective HLA molecules, which again are similar to each other. ${ }^{656}$ The structural differences between the predisposing and protective molecules are reflected by functional differences in (1) peptide selectivity and binding affinity, (2) the interaction with TCRs, and (3) molecular stability on the surface of the antigen presenting cell.

\section{Peptide binding}

The architecture of the antigen binding clefts of the DR and DQ molecules is very similar (fig 4). Both molecules bind to peptides of 12-25 amino acids in length, using conserved residues distributed throughout the binding sites. These form hydrogen bonds with the amino and carbonyl groups along the backbone of the peptide. ${ }^{67}$ Amino acid side chains of the peptide also slot into a series of deep cavities within the binding cleft, termed "pockets". These pockets are highly polymorphic and their structure provides the basis for the "peptide binding motif" of the molecule; that is, the preference for particular amino acid residues at crucial anchor points along the peptide. Structurally distinct HLA molecules favour different peptide binding motifs, ${ }^{68-70}$ dictated by the shape and size of the pockets, and may therefore interact differently with a given antigenic peptide. The key determinants of the binding motif are pockets 1,4 , and 9 (P1, P4, and P9, respectively). ${ }^{65} 66$

P1

In the HLA molecules that predispose to type 1 diabetes, the $\mathrm{Pl}$ pocket is generally much deeper compared with that seen in the protective molecules. For example, $\mathrm{Pl}$ in the predisposing DR4 molecules (encoded by DRB1*0401 or DRB1*0405) contains a glycine residue at position 86 of the $\beta$ chain $(\beta 86)$, which confers a preference for binding to large aromatic side 


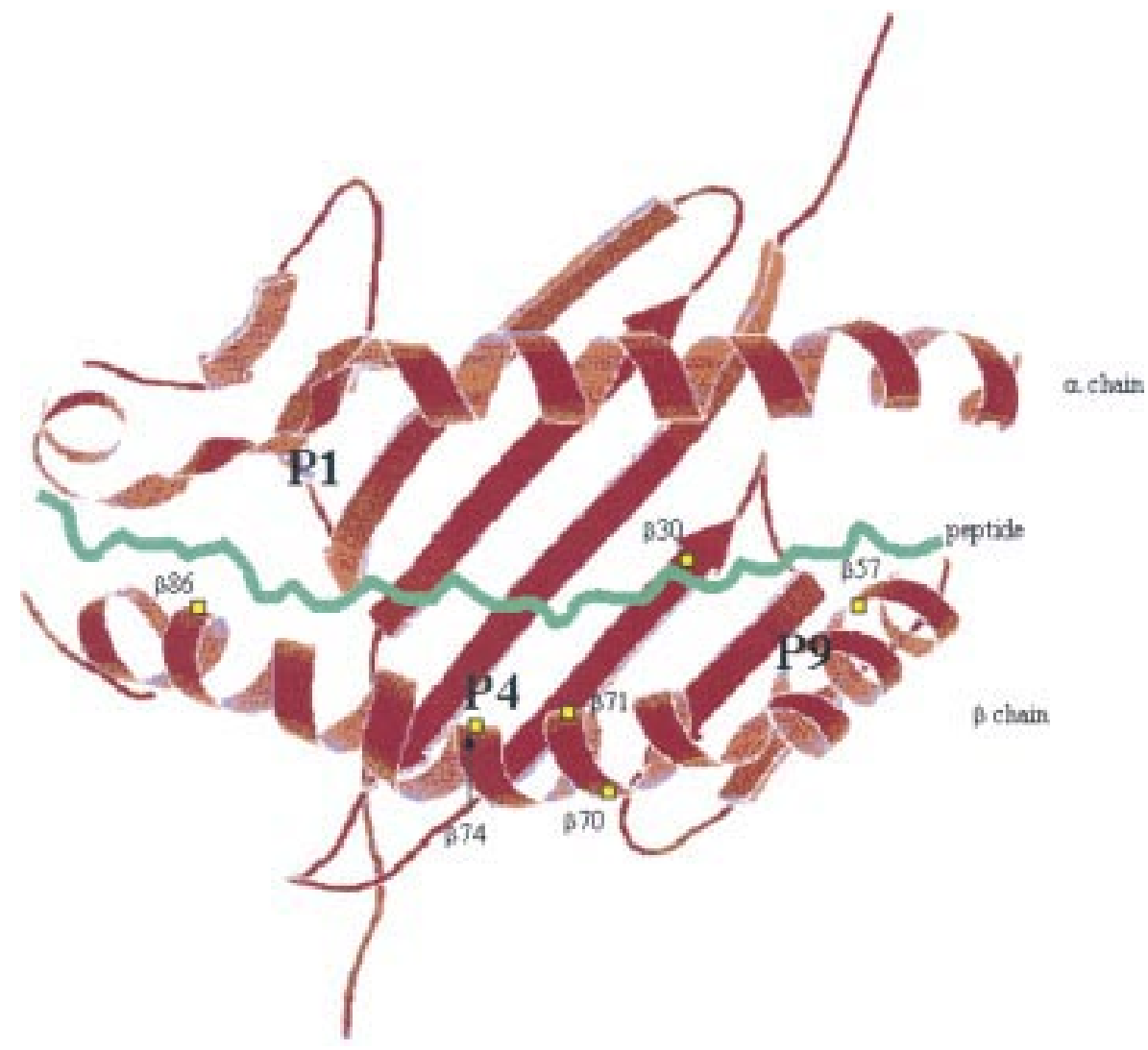

Figure 4 The antigen binding cleft of a human leucocyte antigen class II molecule showing the binding pockets (P1, P4, and P9) and the amino acid residues implicated in peptide binding and T cell activation.

chains in the peptide. In contrast, the valine residue encoded at position $\beta 86$ in the protective DR4 molecule (encoded by DRB $1 * 0403$ ) creates a preference for small or medium sized hydrophobic residues in the peptide. ${ }^{65}$ The $\mathrm{Pl}$ pockets of the predisposing DQ2 and DQ8 molecules are similarly much deeper than that of the protective DQ6.2 molecule. ${ }^{65}$

\section{P4}

The $\mathrm{P} 4$ pocket is an important determinant of peptide binding selectivity in the DR molecule. The alanine residue at position $\beta 74$ in the diabetes permissive DR4 $\left(\mathrm{DRBl}^{*} 0401\right)$ molecule produces a $\mathrm{P} 4$ pocket with a high affinity for acidic residues. In contrast, peptides containing acidic residues at this anchor position are unable to bind to the protective DR4 molecule, encoded by DRB1*0403, because of the presence of an incompatible glutamate residue at position $\beta 74 .{ }^{71}$ The $\mathrm{P} 4$ pocket in the DQ molecule is unlikely to play a crucial role in determining susceptibility to diabetes because its predicted structure is similar in DQ2, DQ8, and DQ6.2. ${ }^{65} 66$

\section{P9}

In general, HLA class II molecules that confer protection against type 1 diabetes carry an aspartate residue at position 57 in the $\beta$ peptide chain $(\operatorname{Asp} \beta 57)$, whereas those that predispose to the disease carry an uncharged amino acid residue at this position (non-Asp $\beta 57$ ), although there are some exceptions to this rule. ${ }^{72-74}$ Residue $\beta 57$ is located within P9, where it plays an important role in determining the structure of this pocket. In molecules carrying Asp $\beta 57$, a salt bridge is formed between this negatively charged residue and a conserved positively charged residue (arginine) at position $\alpha 76$ (in DR molecules) or $\alpha 79$ (in DQ molecules) (fig 5). ${ }^{66} 7576$ This alters the shape of $\mathrm{P} 9$ relative to that seen in non-Asp $\beta 57$ molecules and hence alters the preference of the molecule for particular anchor residues in the bound peptide. Several stud- ies have suggested that the non-Asp $\beta 57$ molecules preferentially bind to peptides with an acidic (negatively charged) residue at the $\mathrm{P} 9$ anchor point, because this residue can form a stabilising salt bridge with the unopposed Arg $\alpha 76$ or Arg $\alpha 79$ residue. ${ }^{66} 6977-79$ However, this does not hold true for the DQ2 molecule, which prefers large hydrophobic residues in P9. ${ }^{80}$ This could be attributed to the neighbouring residues, which produce a larger P9 pocket than that found in other non-Asp $\beta 57$ molecules-for example, DQ8. ${ }^{66}$ This highlights the importance of the morphology of the entire pocket, rather than the influence of a single residue. Nevertheless, the amino acid residue at position $\beta 57$ does have a profound impact on the peptide binding affinity and selectivity of DR and DQ molecules. ${ }^{69} 788182$

In summary, the structural differences between the predisposing and protective HLA molecules may result in differences in their ability to bind to diabetogenic antigens. This may determine whether diabetes develops or not, although the mechanisms involved are unclear.

\section{T cell interaction}

Crystal structures of class II HLA-DR-peptide- $\alpha \beta T C R$ and class I HLA-A-peptide- $\alpha \beta$ TCR complexes have been elucidated. ${ }^{83}{ }^{84}$ These clearly show that different TCRs can bind in a similar orientation to different HLA molecules. The TCR presents a relatively flat surface, which is tilted at an angle to bind to the HLA-peptide complex by avoiding peaks or $\alpha$ helical borders of the antigen binding cleft (fig 3). ${ }^{84}$ The activation of a particular T cell, via its receptor, is influenced by the structure of both the HLA molecule and the peptide being presented.

The $\mathrm{T}$ cell receptor is thought to have several docking points on the HLA class II molecule that are conserved in all DR and DQ heterodimers. In addition, it interacts with some of the polymorphic residues that distinguish one HLA molecule from another. ${ }^{84}$ Thus, the unique combination of amino acid 


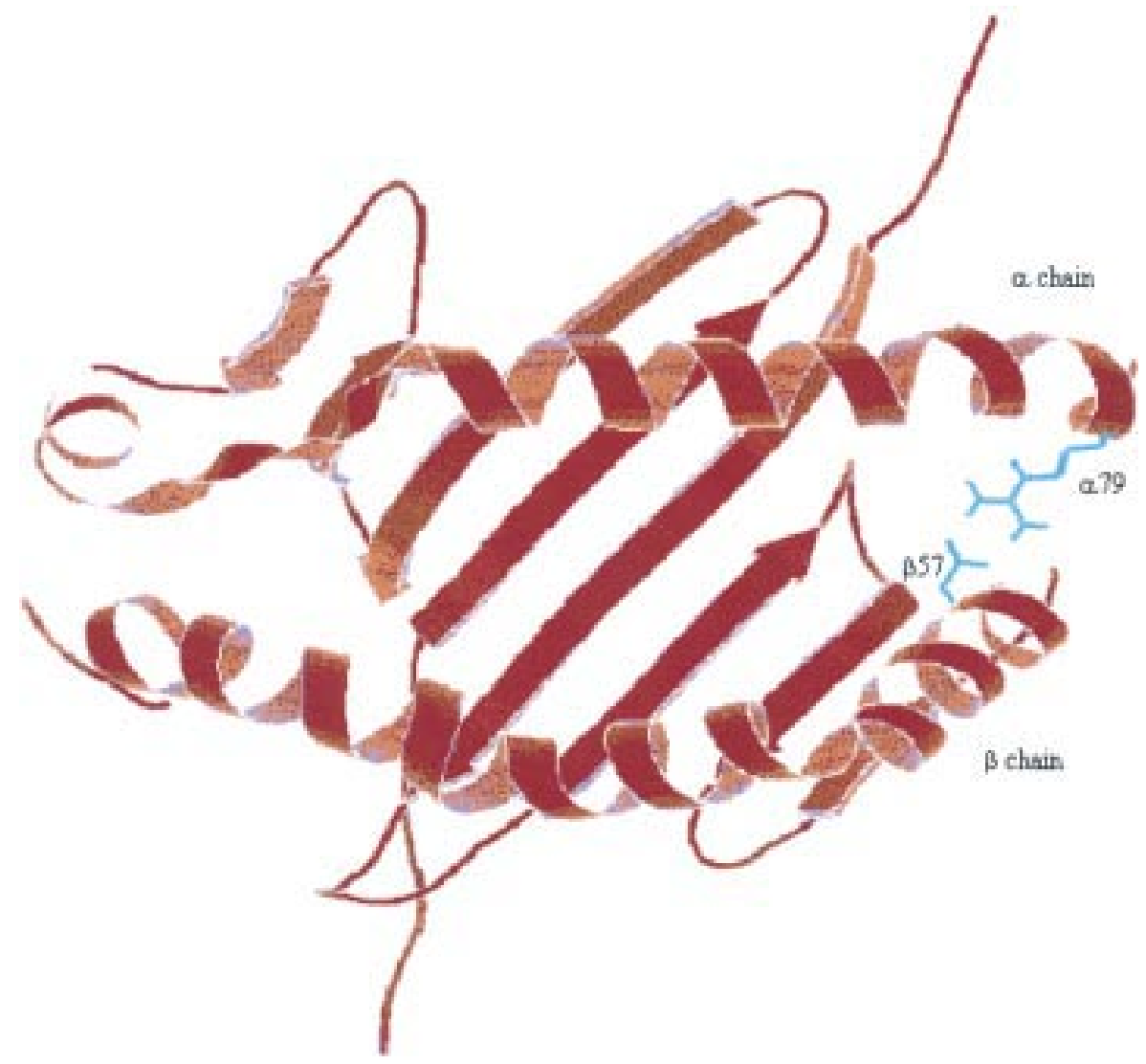

Figure 5 The antigen binding cleft of a human leucocyte antigen class II DQ molecule, showing the salt bridge between residues $\beta 57$ and $\alpha 79$.

residues that characterises the antigen binding cleft of a particular HLA molecule will determine which T cell populations can respond to peptides presented by that molecule. Functional studies of DR and DQ molecules have suggested that residues $\beta 70$ and $\beta 71$ may be crucial contact points for $\mathrm{T}$ cell recognition of the HLA-peptide complex, and that residues $\beta 57$ and $\beta 30$ may also influence T cell activation. ${ }^{85-87}$

"The unique combination of amino acid residues that characterises the antigen binding cleft of a particular HLA molecule will determine which T cell populations can respond to peptides presented by that molecule"

Residues of the peptide presented in the antigen binding cleft are also contacted by the TCR. The amino acid side chains of the peptide residues that bind to the $\mathrm{P} 2, \mathrm{P} 5$, and $\mathrm{P} 8$ pockets in the HLA molecule (termed p2, p5, and p8, respectively) have been suggested to be crucial for T cell activation. ${ }^{88}$ Substitution studies have identified the central residue, p5, as the primary TCR contact point for many peptides. For example, Nepom and colleagues $^{89}$ showed that a conservative substitution at the $\mathrm{p} 5$ residue of a GAD peptide had little effect on binding to the DR4 (DRBI*0401) molecule, but rendered the DR-peptide complex incapable of stimulating GAD specific T cells from patients with diabetes. In contrast, De Oliveira et al showed that TCR interaction with the $\mathrm{p} 5$ residue is not always essential for antigen recognition and can be compensated by interactions with the residues at $\mathrm{p} 2$ and $\mathrm{p} 8{ }^{88}$ Clearly, further research is necessary to understand TCR-peptide-HLA interactions fully.

\section{Molecular stability}

In general, the HLA-DQ molecules associated with protection from type 1 diabetes are more stable on the cell membrane than those associated with susceptibility. ${ }^{90}$ This could lead to an extended half life of "protective" DQ-peptide complexes, which might influence disease risk by altering the strength of the interaction with pathogenically relevant $\mathrm{T}$ cells and ultimately affecting their activation status. The stability of the DQ molecule may be determined, in part, by the amino acid residue at position 57 in the $\beta$ peptide chain. We, and others, have shown that the Asp $\beta 57$ residue is crucial for the stability of the protective DQ6.2 molecule. ${ }^{91}{ }^{92}$ In contrast, however, soluble DQ heterodimers encoded by DQA ${ }^{*} 0201$. DQB ${ }^{*} 0302$ and DQA1*0201.DQB1*0303 were shown to have similar stability, despite differing only at residue $\beta 57$ (Ala versus Asp, respectively).$^{82}$ This suggests that other residues must also play an important role in maintaining the integrity of the class II molecule.

\section{Possible mechanisms by which HLA molecules influence} the development of type 1 diabetes

Clearly, the structural differences seen between the predisposing and protective HLA molecules will affect their ability to interact with diabetogenic antigens and the TCRs of autoreactive, $\beta$ cell specific T cells. Several mechanisms have been proposed to explain how this might influence the risk of developing autoimmune type 1 diabetes. ${ }^{93}$

(1) Antigen binding in the periphery: predisposing HLA molecules may bind well to diabetogenic antigens in the periphery and hence activate an autoimmune $\mathrm{T}$ cell response, whereas protective HLA molecules may not. Alternatively, the protective molecules may bind to the autoantigens with a higher affinity, thus competing with the predisposing molecules. In this last scenario, the threshold of binding required for $\mathrm{T}$ cell activation restricted by the predisposing molecules may not be reached. 
(2) Molecular stability and thymic deletion of autoreactive T cells: protective HLA molecules may form stable complexes with self antigens in the thymus, leading to efficient deletion of potentially autoreactive $\mathrm{T}$ cells. In contrast, the less stable complexes formed by the predisposing HLA molecules may result in inefficient $\mathrm{T}$ cell removal and the release of autoreactive $\mathrm{T}$ cells into the periphery. Negative selection of diabetogenic $\mathrm{T}$ cells by protective class II molecules has been demonstrated in one mouse model, ${ }^{94}$ but not in another. ${ }^{95}$

(3) Influence on T cell phenotype: predisposing and protective HLA molecules may interact differently with the TCRs of autoreactive $\mathrm{T}$ cells, affecting the phenotype of the $\mathrm{T}$ cells (proinflammatory versus regulatory) or their activation status (proliferative versus anergised). This immunomodulatory hypothesis is supported by the observation that DQ6.2 can protect against the development of diabetes, even after the onset of $\beta$ cell autoimmunity. ${ }^{56}$

It is unclear exactly which mechanisms are involved in determining the risk of type 1 diabetes. Currently, functional studies aiming to deal with this question are limited because the antigenic peptide(s) and T cell populations involved in initiating and perpetuating the autoimmune attack on the $\beta$ cells are yet to be identified. Advances in understanding the environmental influences on disease development may be necessary before progress can be made in identifying the crucial components of $\beta$ cell destruction. To identify pathogenically relevant $\mathrm{T}$ cell populations, it may be necessary to study individuals at the onset of the autoimmune attack, rather than at the time of clinical diagnosis, when such $\mathrm{T}$ cells may no longer be present. Suitable prediabetic subjects for these studies could be recruited from among first degree relatives of diabetic probands, although intensive monitoring would be necessary to determine the onset of $\beta$ cell autoimmunity.

\section{IDDM2: THE INSULIN GENE VNTR AND TYPE 1 DIABETES}

Several studies of white populations have shown an association between type 1 diabetes and polymorphisms within the insulin gene region on chromosome 1lp15.5..$^{3596}$ The primary association is thought to be with a variable number of tandem repeats (VNTR) region, located $596 \mathrm{bp}$ upstream of the translational start site of the insulin gene. ${ }^{97}$ Three classes of VNTR alleles have been identified, segregated according to the number of repeats of a 14-15 bp sequence: class I alleles (20-63 repeats), class II alleles (64-139 repeats), and class III alleles ( $140-210$ repeats).

The class I alleles are generally associated with susceptibility to type 1 diabetes, with the highest risk conferred by class I homozygosity, whereas the class III alleles are associated with dominant protection, ${ }^{97}$ although there are exceptions to this pattern for both class I and class III alleles. Some class I alleles are not predisposing. ${ }^{97}{ }^{98}$ It has recently been suggested that the class I alleles can be subdivided into three groups, termed IC +, ID +, and ID-, based on a combination of variant repeat distributions and flanking haplotypes. All class I alleles are equally predisposing to diabetes except the ID- alleles, which are protective when transmitted from class I ID-/class III heterozygous fathers. ${ }^{99}$ The class III alleles can also be subdivided into two highly diverged lineages, designated IIIA and IIIB. ${ }^{99}$ The class IIIA alleles are protective against type 1 diabetes, whereas the class IIIB alleles are very protective. A recent study also identified a subgroup of rare class III alleles that appear to predispose to diabetes. ${ }^{100}$

\section{Functional relevance of the insulin VNTR and diabetes susceptibility}

The mechanism by which the insulin VNTR polymorphisms influence the risk of type 1 diabetes is unclear. However, this locus has been shown to regulate the expression of two downstream genes that may be relevant to disease pathogenesis, namely: the insulin gene and the insulin-like growth factor 2 (IGF2) gene.

Insulin and its precursors are potential target autoantigens for $\beta$ cell destruction. Transcripts of the insulin gene have been detected in the human thymus, in addition to pancreatic islets, and the amounts of insulin mRNA are reported to correlate with allelic variation at the VNTR locus. Protective class III alleles are associated with increased transcription of the insulin gene in the thymus compared with predisposing class I alleles. In contrast, higher amounts of insulin mRNA in the pancreas are associated with the predisposing class I alleles. ${ }^{101-103}$ Raised concentrations of preproinsulin in the thymus may promote the efficient deletion of autoreactive T cells specific for this protein, leading to immune tolerance to a key autoantigen in the pathogenesis of diabetes. This mechanism may explain the dominant protective effect of the class III VNTR alleles. In subjects homozygous for the predisposing class I alleles, the combination of lower intrathymic insulin expression and higher expression in the pancreas would be expected to increase the risk of insulin driven pancreatic autoimmunity. This immune tolerance hypothesis is supported by the finding of two rare class III alleles, which are associated with complete silencing of thymic insulin transcripts and are reported to predispose to type 1 diabetes. ${ }^{100}$ Furthermore, a recent study of a mouse model in which there was graded thymic insulin deficiency showed an inverse correlation between thymic insulin concentrations and peripheral $\mathrm{T}$ cell reactivity to insulin. ${ }^{104}$ To date, however, there is no evidence of a correlation between insulin VNTR class and induction of tolerance to insulin in humans. ${ }^{105}$

"Raised concentrations of preproinsulin in the thymus may promote the efficient deletion of autoreactive $T$ cells specific for this protein, leading to immune tolerance to a key autoantigen in the pathogenesis of diabetes"

The IGF2 gene product (IGF-II) may also contribute to IDDM2 associated susceptibility to diabetes. Thymic IGF-II has been suggested to influence the risk of pancreatic autoimmunity, because it plays an important role in $\mathrm{T}$ cell development and negative selection. It may also act as a selecting peptide for insulin reactive T cells, as a result of its homology to proinsulin. ${ }^{106}$ This mechanism is unlikely to account for the disease associations observed with the insulin VNTR alleles, however, because the class I and class III alleles are associated with similar levels of IGF2 expression in the pancreas and thymus. ${ }^{106} 107$ However, the predisposing class I alleles are associated with increased expression of IGF2 in the placenta. ${ }^{108}$ This has been suggested to influence intrauterine growth and birth size, which are both risk factors for type 1 diabetes. ${ }^{109}$

\section{SUMMARY}

The HLA genes are the strongest genetic determinants for type $\mathrm{l}$ diabetes identified to date, and the insulin VNTR also makes a contribution to disease risk. Although these risk factors are fairly well characterised at the genetic level, it is still unclear exactly how they influence susceptibility to the disease. Further functional studies are required to rectify this lack of knowledge. However, studies aiming to elucidate the mechanism by which HLA molecules influence disease risk are currently hampered by a lack of knowledge regarding the key autoantigenic peptide(s) and T cell populations responsible for the initiation and amplification of $\beta$ cell destruction. Despite considerable research work, these vital components are yet to be identified. The influence of environmental susceptibility factors also remains unclear. Further research work aimed at identifying susceptibility determinants (both genetic and 


\section{Take home messages}

- Type 1 diabetes is a T cell mediated autoimmune disease, characterised by the selective destruction of pancreatic $\beta$ cells

- Susceptibility to the disease is determined by a combination of genetic and environmental factors

- Several environmental agents have been implicated in disease risk, including viruses and dietary factors, although none has yet been shown to be directly responsible for triggering $\beta$ cell autoimmunity

- The genetic factors that influence disease risk have been subjected to more intensive study and over 20 chromosomal regions have been reported to contain susceptibility genes for type 1 diabetes

- Of these, only two loci have been well characterised: the human leucocyte antigen (HLA) locus and the insulin gene

- The HLA class II genes, DRB 1 and DQB1, provide the strongest genetic risk component, although their influence may be modified by other genes within the major histocompatibility complex

- The molecules encoded by the DR and $D Q$ genes are thought to play a central role in the thymic selection and subsequent activation of the autoreactive T cells responsible for $\beta$ cell destruction, and their influence on disease risk is probably related to the presence of specific structural features, which determine the ability of the HLA molecules to interact with diabetogenic T cell populations

- The variable number of tandem repeats (VNTR) region located upstream of the insulin gene also makes an important contribution to diabetes susceptibility. The molecular mechanism involved is unknown although it may be related to the selection/deletion of insulin specific $T$ cells in the thymus

- Further research is necessary to elucidate the precise mechanisms by which these genetic factors influence the risk of developing type 1 diabetes and to determine how they might interact with environmental susceptibility factors

- Such knowledge will further our understanding of the molecular pathology of the disease and may contribute to the design of novel therapeutic strategies

environmental) may benefit from better definition of disease phenotype, because disease heterogeneity is a significant confounding factor in such studies. Although advances may be slow, it is nevertheless important to improve our understanding of the molecular pathology of type 1 diabetes and the role played by the major susceptibility factors. This knowledge will facilitate the development of novel treatment strategies, aimed at preventing the onset and/or progression of the autoimmune process.

\section{Authors' affiliations}

M A Kelly, M L Rayner, C H Mijovic, A H Barnett, Department of Medicine, Division of Medical Sciences, University of Birmingham and Birmingham Heartlands Hospital, Birmingham B9 5SS, UK

\section{REFERENCES}

1 Couper JJ, Hudson I, Werther GA, et al. Factors predicting residual beta-cell function in the first year after diagnosis of childhood type 1 diabetes. Diabetes Res Clin Pract 1991;11:9-16.

2 Gepts W. Pathologic anatomy of the pancreas in juvenile diabetes. Diabetes 1965;14:619-33.

3 Gepts W, De Mey J. Islet cell survival determined by morphology: an immunocytochemical study of the islets of Langerhans in juvenile diabetes mellitus. Diabetes 1978;27:251-61.

4 MacCuish AC, Jordan J, Campbell CJ, et al. Cell mediated immunity in diabetes mellitus: lymphocyte transformation by insulin and insulin fragments in insulin treated and newly diagnosed diabetics. Diabetes $1975 ; 24: 36-43$

5 Naquet $\mathbf{P}$, Ellis J, Tibensky D, et al. T cell autoreactivity to insulin in diabetic and related non-diabetic individuals. J Immunol 1988; 140:2569-78

6 Atkinson MA, Kaufman DL, Campbell L, et al. Response of peripheral-blood mononuclear cells to glutamate decarboxylase in insulin-dependent diabetes. Lancet 1992;339:458-9.
7 Lohmann T, Leslie RDG, Hawa M, et al. Immunodominant epitopes of glutamic acid decarboxylase 65 and 67 in insulin-dependent diabetes mellitus. Lancet 1994;343:1607-8.

8 Hawkes CJ, Schloot NC, Marks J, et al. T-cell lines reactive to an immunodominant epitope of the tyrosine phosphatase-like autoantigen IA-2 in type 1 diabetes. Diabetes 2000;49:356-66.

9 Boitard C, Debray-Sachs M, Poulard A, et al. Lymphocytes from diabetics suppress insulin release in vitro. Diabetologia 1981;21:41-6.

10 Todd JA, Aitman TJ, Cornall RJ, et al. Genetic analysis of autoimmune type 1 diabetes mellitus in mice. Nature 1991;351:542-7.

11 Wicker LS, Miller BJ, Mullen Y. Transfer of autoimmune diabetes mellitus with splenocytes from nonobese diabetic (NOD) mice. Diabetes 1986;35:855-60.

12 Bendelac A, Carnaud C, Boitard C, et al. Syngeneic transfer of autoimmune diabetes from diabetic NOD mice to healthy neonates. Requirement for both L3T4+ and Lyt-2+ T cells. J Exp Med 1987; 166:823-32

13 Rohane PW, Shimada A, Kim DT, et al. Islet-infiltrating lymphocytes from prediabetic NOD mice rapidly transfer diabetes to NOD-scid/scid mice. Diabetes 1995;44:550-4

14 Leslie RD, Atkinson MA, Notkins AL. Autoantigens IA-2 and GAD in type 1 (insulin-dependent) diabetes. Diabetologia 1999;42:3-14.

15 Palmer JP, Asplin CM, Clemons P, et al. Insulin antibodies in insulin-dependent diabetics before insulin treatment. Science 1983;222: 1337-9.

16 Ziegler AG, Hummel M, Schenker M, et al. Autoantibody appearance and risk for development of childhood diabetes in offspring of parents with type 1 diabetes: the 2 year analysis of the German BABYDIAB study. Diabetes 1999;48:460-8.

17 Bingley PJ, Bonifacio E, Williams AJK, et al. Prediction of IDDM in the general population. Strategies based on combinations of autoantibody markers. Diabetes 1997;46:1701-10.

18 LaGasse JM, Brantley MS, Leech NJ, et al. Successful prospective prediction of type 1 diabetes in schoolchildren through multiple defined autoantibodies: an 8-year follow-up of the Washington State diabetes prediction study. Diabetes Care 2002;25:505-11.

19 Risch N. Assessing the role of HLA-linked and unlinked determinants of disease. Am J Hum Genet 1987;40: 1-14.

20 Olmos $P, A^{\prime}$ Hern R, Heaton DA, et al. The significance of the concordance rate for type 1 (insulin-dependent) diabetes in identical twins. Diabetologia 1988;31:747-50.

21 Redondo MJ, Yu L, Hawa M, et al. Heterogeneity of type 1 diabetes: analysis of monozygotic twins in Great Britain and the United States. Diabetologia 2001;44:354-62.

22 Gamble DR, Taylor KW. Seasonal incidence of diabetes mellitus. BM 1969:3:631-3.

23 Siemiatycki J, Colle E, Campbell S, et al. Incidence of IDDM in Montreal by ethnic group and by social class and comparisons with ethnic groups living elsewhere. Diabetes 1988;37:1096-102.

24 Knip M, Akerblom HK. Environmental factors in the pathogenesis of type 1 diabetes mellitus. Exp Clin Endocrinol Diabetes 1999;107:S93-100.

25 Ellis TM, Atkinson MA. Early infant diets and insulin-dependent diabetes. Lancet 1996;347:1464-5.

26 Dahlquist GG. Viruses and other perinatal exposures as initiating events for beta-cell destruction. Ann Med 1997;29:413-17

27 Dahlquist GG, Patterson C, Soltesz G. Infections and vaccinations as risk factors for childhood type 1 (insulin-dependent) diabetes mellitus: a multicentre case-control investigation. EURODIAB substudy 2 study group. Diabetologia 2000;43:47-53

28 Wasmuth HE, Hess G, Viergutz C, et al. Non-specific viral infections as possible synchronizing events of the manifestation of type 1 diabetes. Diabetes Metab Res Rev 2000;16:177-8.

29 McKinney PA, Parslow R, Gurney KA, et al. Perinatal and neonatal determinants of childhood type 1 diabetes: a case-control study in Yorkshire. Diabetes Care 1999;22:928-32.

30 Ou D, Mitchell LA, Metzger DL, et al. Cross-reactive rubella virus and glutamic acid decarboxylase (65 and 67) protein determinants recognised by $T$ cells of patients with type 1 diabetes mellitus. Diabetologia 2000;43:750-62.

31 Varela-Calvino R, Ellis R, Sgarbi G, et al. Characterization of the T-cell response to coxsackie B4: evidence that effector memory cells predominate in patients with type 1 diabetes. Diabetes 2002;51:1745-53.

32 Singal DP, Blajchman MA. Histocompatibility $(\mathrm{HL}-\mathrm{A})$ antigens, lymphocytotoxic antibodies and tissue antibodies in patients with diabetes mellitus. Diabetes 1973;22:429-32.

33 Nerup J, Platz P, Andersen OO, et al. HL-A antigens and diabetes mellitus. Lancet 1974; ii:864-6.

34 Cudworth AG, Woodrow JC. HL-A antigens and diabetes mellitus. Lancet 1974;ii: 1153.

35 Bell GI, Horita S, Karam JH. A polymorphic locus near the human insulin gene is associated with insulin-dependent diabetes mellitus. Diabetes 1984;33:176-83.

36 Bain SC, Prins JB, Hearne CM, et al. Insulin gene region-encoded susceptibility to type 1 diabetes is not restricted to HLA-DR4-positive individuals. Nat Genet 1992;2:212-15.

37 Davies JL, Kawaguchi Y, Bennett ST, et al. A genome-wide search for human type 1 diabetes susceptibility genes. Nature 1994;371:130-6.

38 Mein CA, Esposito L, Dunn MG, et al. A search for type 1 diabetes susceptibility genes in families from the United Kingdom. Nat Genet 1998; 19:297-300.

39 Concannon P, Gogolin-Ewens KJ, Hinds DA, et al. A second-generation screen of the human genome for susceptibility to insulin-dependent diabetes mellitus. Nat Genet 1998;19:292-6. 
40 Cox NJ, Wapelhorst B, Morrison VA, et al. Seven regions of the genome show evidence of linkage to type 1 diabetes in a consensus analysis of 767 multiplex families. Am J Hum Genet 2001;69:820-30.

41 Spielman RS, McGinnis RE, Ewens WJ. Transmission test for linkage disequilibrium: the insulin gene region and insulin-dependent diabetes mellitus (IDDM). Am J Hum Genet 1993:52:506-16.

42 Luo DF, Buzzetti R, Rotter Jl, et al. Confirmation of three susceptibility genes to insulin-dependent diabetes mellitus: IDDM4, IDDM5 and IDDM8. Hum Mol Genet 1996;5:693-8

43 She JX, Marron MP. Genetic susceptibility factors in type 1 diabetes: linkage, disequilibrium and functional analyses. Curr Opin Immunol 1998;10:682-9.

44 Merriman T, Twells R, Merriman M, et al. Evidence by allelic association-dependent methods for a type 1 diabetes polygene (IDDM6) on chromosome 18q21. Hum Mol Genet 1997;6:1003-10.

45 Herr M, Dudbridge F, Zavattari $P$, et al. Evaluation of fine mapping strategies for a multifactorial disease locus: systematic linkage and association analysis of IDDM1 in the HLA region of chromosome 6p21. Hum Mol Genet 2000; 9:1291-301.

46 Nejentsev S, Reijonen H, Adojaan B, et al. The effects of HLA-B allele on the IDDM risk defined by DRB I ${ }^{*} 04$ subtypes and DQB 1 *0302. Diabetes 1997:46: 1888-92

47 Noble JA, Valdes AM, Thomson G, et al. The HLA class II locus DPB 1 can influence susceptibility to type 1 diabetes. Diabetes 2000;49:121-5.

48 She JX. Susceptibility to type 1 diabetes: HLA-DQ and DR revisited. Immunol Today 1996:17:323-9.

49 Kockum I, Sanjeevi CB, Eastman S, et al. Complex interaction between HLA DR and DQ in conferring risk for childhood type 1 diabetes. Eur J Immunogenet 1999;26:361-72.

50 Donner H, Seidl C, van der Auwera B, et al. HLA-DRB 1 *04 and susceptibility to type 1 diabetes mellitus in a German/Belgian family and German case/control study. Tissue Antigens 2000;55:271-4.

51 Undlien DE, Friede T, Rammensee HG, et al. HLA-encoded genetic predisposition in IDDM. DR4 subtypes may be associated with different degrees of protection. Diabetes 1997:46:143-9.

52 Roep BO, Schipper R, Verduyn W, et al. HLA-DRB 1 *0403 is associated with dominant protection against IDDM in the general Dutch population and subjects with high-risk DQA 1 *0301-DQB 1 *0302/ DQA $1 * 0501-D Q B 1 * 0201$ genotype. Tissue Antigens 1999;54:88-90.

53 van der Auwera B, van Waeyenberge $C$, Schuit F, et al. DRB 1 *0403 protects against IDDM in Caucasians with the high-risk heterozygous DQA 1 *0301-DQB 1 *0302/DQA 1 *0501-DQB 1 *0201 genotype. Diabetes 1995;44:527-30

54 Redondo MJ, Kawasaki E, Mulgrew CL, et al. DR-and DQ-associated protection from type 1A diabetes: comparison of DRB 1 *1401 and $\mathrm{DQA1}$ *0102-DQB1 *0602. J Clin Endocrinol Metab 2000;85:3793-7.

55 Baisch JM, Weeks T, Giles R, et al. Analysis of HLA-DQ genotypes and susceptibility in insulin-dependent diabetes mellitus. N Engl J Med 1990;322:1836-41

56 Pugliese A, Gianani R, Moromisato R, et al. HLA-DQB 1 *0602 is associated with dominant protection from diabetes even among islet cell antibody-positive first-degree relatives of patients with IDDM. Diabetes 1995;44:608-13.

57 Pugliese A, Kawasaki E, Zeller M, et al. Sequence analysis of the diabetes-protective human leukocyte antigen-DQB 1*0602 allele in unaffected, islet cell antibody-positive first degree relatives and in rare patients with type 1 diabetes. J Clin Endocrinol Metab patients with type 1

58 Rayner ML, Kelly MA, Mijovic $\mathrm{CH}$, et al. Sequencing of the second exon of the MHC class II DQ6 alleles in patients with type 1 diabetes. Autoimmunity 2002;35:155-7.

59 Undlien DE, Kockum I, Ronningen KS, et al. HLA associations in type 1 diabetes among patients not carrying high-risk DR3-DQ2 or DR4-DQ8 haplotypes. Tissue Antigens 1999;54:543-51.

60 Mijovic $C$, Jenkins $D$, Jacobs $K$, et al. HLA-DQA 1 and DQB 1 alleles are associated with genetic susceptibility to type 1 diabetes in a Black population. Diabetes 1991;40:748-53

61 Kawasaki E, Noble J, Erlich $\mathrm{H}$, et al. Transmission of DQ haplotypes to patients with type 1 diabetes. Diabetes 1998;47:1971-3.

62 Abraham RS, Kudva YC, Wilson SB, et al. Co-expression of HLA DR3 and DQ8 results in the development of spontaneous insulitis and loss of tolerance to GAD 65 in transgenic mice. Diabetes 2000;49:548-54.

63 Abraham RS, Wen L, Marietta EV, et al. Type 1 diabetes-predisposing MHC alleles influence the selection of glutamic acid decarboxylase (GAD) 65-specific T cells in a transgenic model. J Immunol 2001;166:1370-9.

64 Wen L, Chen N-Y, Tang J, et al. The regulatory role of DR4 in a spontaneous diabetes DQ8 transgenic model. J Clin Invest 2001;107:871-80.

65 Cucca $F$, Lampis $R$, Congia $M$, et al. A correlation between the relative predisposition of MHC class II alleles to type 1 diabetes and the structure of their proteins. Hum Mol Genet 2001; 10:2025-37.

66 Lee KH, Wucherpfennig KW, Wiley DC. Structure of a human insulin peptide-HLA-DQ8 complex and susceptibility to type 1 diabetes. Nat Immunol 2001;2:501-7.

67 Brown JH, Jardetzky T, Saper MA, et al. A hypothetical model of the foreign antigen binding site of class II histocompatibility molecules. Nature 1988;332:845-50.

68 Ettinger RA, Kwok WW. A peptide binding motif for HLA-DQA 1 *0102/DQB 1 *0602, the class II MHC molecule associated with dominant protection in insulin-dependent diabetes mellitus. J Immunol 1998; 160:2365-73
69 Kwok WW, Domeier ME, Raymond FC, et al. Allele-specific motifs characterize HLA-DQ interactions with a diabetes-associated peptide derived from glutamic acid decarboxylase. J Immunol 1996;156:21717 .

70 Vartdal $\mathbf{F}$, Johansen $\mathrm{BH}$, Friede $\mathrm{T}$, et al. The peptide binding motif of the disease associated HLA-DQ (alpha 1*0501, beta 1*0201) molecule. Eur J Immunol 1996;26:2764-72.

71 Dessen A, Lawrence CM, Cupo $S$ et al. X ray crystal structure of HLA-DR4 (DRA*0101,DRB 1 *0401) complexed with a peptide from human collagen II. Immunity 1997;7:473-81.

72 Todd JA, Bell JI, McDevitt HO. HLA-DQ beta gene contributes to susceptibility and resistance to insulin-dependent diabetes mellitus. Nature 1987;329:599-604.

73 Nepom GT, Erlich H. MHC class-II molecules and autoimmunity. Annu Rev Immunol 1991;9:493-525

74 Awata T, Kuzuya T, Matsuda A, et al. High frequency of aspartic acid at position 57 of HLA-DQ $\beta$ chain in Japanese IDDM patients and non-diabetic subjects. Diabetes 1990;39:266-9.

75 Brown JH, Jardetzky TS, Gorga JC, et al. Three-dimensional structure of the human class II histocompatibility antigen HLA-DR 1. Nature 1993; 364:33-9.

76 Stern LJ, Brown JH, Jardetzky TS, et al. Crystal structure of the human class II MHC protein HLA-DR 1 complexed with an influenza virus peptide. Nature 1994;368:215-21.

77 Reizis B, Eisenstein M, Bockova J, et al. Molecular characterization of the diabetes-associated mouse MHC class II protein, I-Ag7. Int Immunol 1997:9:43-51.

78 Kwok WW, Domeier ME, Johnson ML, et al. HLA-DQB 1 codon 57 is critical for peptide binding and recognition. J Exp Med 1996; 183:1253-8

79 Kinouchi R, Kobayasi H, Sato K, et al. Peptide motifs of HLA-DR4/DR53 (DRB1 *0405/DRB4*0101) molecules. Immunogenetics 1994;40:376-8.

80 Quarsten H, Paulsen G, Johansen BH, et al. The P9 pocket of HLA-DQ2 (non-Aspbeta57) has no particular preference for negatively charged anchor residues found in other type 1 diabetes-predisposing non-Aspbeta57 MHC class II molecules. Int Immunol 1998;10:1229-36.

81 Nepom BS, Nepom GT, Coleman M, et al. Critical contribution of $\beta$ chain residue 57 in peptide binding ability of both HLA-DR and -DQ molecules. Proc Natl Acad Sci U S A 1996;93:7202-6.

82 Sato AK, Sturniolo T, Sinigaglia F, et al. Substitution of aspartic acid at $\beta 57$ with alanine alters $M H C$ class II peptide binding activity but not protein stability: $\left.\mathrm{HLA}-\mathrm{DQ}(\alpha)^{*} 0201, \beta 1{ }^{*} 0302\right)$ and $(\alpha)^{*} 0201$ $\beta 1 * 0303)$. Hum Immunol 1999;60:1227-36.

83 Garboczi DN, Ghosh P, Utz U, et al. Structure of the complex between human T-cell receptor, viral peptide and HLA-A2. Nature 1996;384:34-41.

84 Hennecke J, Carfi A, Wiley DC. Structure of a covalently stabilized complex of a human alphabeta T-cell receptor, influenza HA peptide and MHC class II molecule, HLA-DR 1. EMBO J 2000; 19:561 1-24.

85 Signorelli KL, Watts LM, Lambert LE. The importance of DR4Dw4 beta chain residues 70,71 and 86 in peptide binding and T cell recognition. Cell Immunol 1995;162:217-24.

86 Coppin HL, Carmichael P, Lombardi G et al. Position 71 in the alpha helix of the DR beta domain is predicted to influence peptide binding and plays a central role in allorecognition. Eur J Immunol 1993;23:343-9.

87 Reichstetter S, Papadopoulos GK, Moustakas AK, et al. Mutational analysis of critical residues determining antigen presentation and activation of HLA-DQ0602 restricted T-cell clones. Hum Immunol 2002;63:185-93

88 De Oliveira DB, Harfouch-Hammoud E, Otto H, et al. Structural analysis of two HLA-DR-presented autoantigenic epitopes: crucial role of peripheral but not central peptide residues for T-cell receptor recognition. Mol Immunol 2001;37:813-25.

89 Nepom GT, Lippolis JD, White FM, et al. Identification and modulation of a naturally processed T cell epitope from the diabetes-associated autoantigen human glutamic acid decarboxylase 65 (hGAD65). Proc Natl Acad Sci U S A 2001;98:1763-8.

90 Ettinger RA, Liu AW, Nepom GT, et al. Exceptional stability of the HLA-DQA 1 *0102/DQB 1 *0602 $\alpha \beta$ protein dimer, the class II MHC molecule associated with protection from insulin-dependent diabetes mellitus. J Immunol 1998;161:439-45.

91 Rayner ML, Mijovic CH, Barnett AH, et al. Aspartate at residue $\beta 57$ is important for the stability and antigen-binding ability of the DQ6.2 molecule. Diabet Med 2002;19:P154.

92 Ettinger RA, Liu AW, Nepom GT, et al. $\beta 57-A s p$ plays an essential role in the unique SDS stability of HLA-DQA ${ }^{*} 0102 / D Q B 1 * 0602 \alpha \beta$ protein dimer, the class II MHC allele associated with protection from insulin-dependent diabetes mellitus. J Immunol 2000;165:3232-8.

93 Nepom GT, Kwok WW. Molecular basis for HLA-DQ associations with IDDM. Diabetes 1998:47:1 177-84.

94 Schmidt D, Verdaguer J, Averill N, et al. A mechanism for the major histocompatibility complex-linked resistance to autoimmunity. J Exp Med 1997; 186: 1059-75.

95 Luhder F, Katz J, Benoist C, et al. Major histocompatibility complex class II molecules can protect from diabetes by positively selecting $T$ cells with additional specificities. J Exp Med 1998;187:379-87.

96 Lucassen AM, Julier C, Beressi JP, et al. Susceptibility to insulin dependent diabetes mellitus maps to a $4.1 \mathrm{~kb}$ segment of DNA spanning the insulin gene and associated VNTR. Nat Genet 1993;4:305-10.

97 Bennett ST, Lucassen AM, Gough SCL, et al. Susceptibility to human type 1 diabetes at IDDM2 is determined by tandem repeat variation at the insulin gene minisatellite locus. Nat Genet 1995;9:284-92. 
98 Bennett ST, Wilson AJ, Esposito L, et al. Insulin VNTR allele-specific effect in type 1 diabetes depends on identity of untransmitted paternal haplotype. Nat Genet 1997;17:350-2

99 Stead JDH, Buard J, Todd JA, et al. Influence of allele lineage on the role of the insulin minisatellite in susceptibility to type 1 diabetes. Hum Mol Genet 2000:9:2929-35.

100 Vafiadis $\mathbf{P}$, Ounissi-Benkalha $\mathrm{H}$, Palumbo $M$, et al. Class III alleles of the variable number of tandem repeat insulin polymorphism associated with silencing of thymic insulin predispose to type 1 diabetes. J Clin Endocrinol Metab 2001:86:3705-10.

101 Vafiadis $\mathbf{P}$, Bennett ST, Colle E, et al. Imprinted and genotype-specific expression of genes at the IDDM2 locus in pancreas and leucocytes. $J$ Autoimmun 1996;9:397-403.

102 Vafiadis $\mathbf{P}$, Bennett ST, Todd JA, et al. Insulin expression in human thymus is modulated by INS VNTR alleles at the IDDM2 locus. Nat Genet 1997; 15:289-92.

103 Pugliese A, Zeller M, Fernandez A, et al. The insulin gene is transcribed in the human thymus and transcription levels correlated with allelic variation at the INS VNTR-IDDM2 susceptibility locus for type 1 diabetes. Nat Genet 1997; 15:293-7.
104 Chentoufi AA, Polychronakos C. Insulin expression levels in the thymus modulate insulin-specific autoreactive T-cell tolerance. The mechanism by which the IDDM2 locus may predispose to diabetes. Diabetes 2002;51:1383-90

105 Sarugeri E, Dozio N, Belloni $C$, et al. Autoimmune responses to the $\beta$ cell autoantigen, insulin, and the INS VNTR-IDDM2 locus. Clin Exp Immunol 1998; 1 14:370-6.

106 Vafiadis P, Grabs R, Goodyer CG, et al. A functional analysis of the role of IGF2 in IDDM2-encoded susceptibility to type 1 diabetes. Diabetes 1998;47:831-6.

107 Vafiadis P, Bennett ST, Todd JA, et al. Divergence between genetic determinants of IGF2 transcription levels in leukocytes and of IDDM2-encoded susceptibility to type 1 diabetes. J Clin Endocrinol Metab 1998;83:2933-9.

108 Paquette J, Giannoukakis N, Polychronakos C, et al. The INS 5' variable number of tandem repeats is associated with IGF2 expression in humans. J Biol Chem 1998;273:14158-64

109 Dahlquist G, Bennich SS, Kallen B. Intrauterine growth pattern and risk of childhood onset insulin dependent (type 1) diabetes: population based case-control study. BM 1996;313:1174-7.

\section{Enzymes aid invasion of arthritic joints}

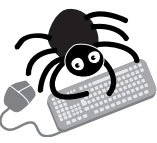

Please visit the Molecular Pathology website [www molpath.com] for link to this full article.
D ecent evidence suggests that enzymes which destroy cartilage aid invasive growth of fibroblast-like synoviocytes (FLS) in rheumatoid arthritis (RA). An in vitro study has compared potential invasive - properties of FLS from patients with RA, osteoarthritis (OA), and avascular necrosis (AVN) and found that FLS from RA had significantly more matrix metalloproteinases (MMPs).

Growth through an artificial matrix was greater for FLS from RA (median cell number $4788 v 1875$ for $\mathrm{OA}, v 1530$ for AVN), and so was growth rate ( 0.27 /day $v 0.22$ /day $v 0.25$ /day, respectively). However, growth rate showed no correlation with cell number.

FLS expressing MMP-1, MMP-3, or MMP-10 were significantly more invasive (median number of invasive cells 3835,4248, and 4990, respectively) whether from RA or OA. But the odds of having MMP-1 and MMP-9 and RA were significant, 6.5 and 10.7, when compared with OA. Other attributesexpression of cathepsin-K and tissue inhibitors of MMP-1 and MMP-2-did not influence invasiveness.

FLS were cultured from tissue obtained from joint replacements or synovectomy in patients with RA (30), OA (17), and AVN (nine). Invasiveness was assayed in a Matrigel transwell culture system, by counting cells that migrated through the matrix after three days' incubation. Growth rate was determined from cell counts of cultures harvested at intervals after seeding. Expression of cathepsin-K, tissue inhibitors, and MMPs was indicated by reverse transcriptase-PCR.

Activated FLS invade the synovium, articular cartilage, and bone in RA. Whether this is through increased growth or invasiveness has not been studied directly before now.

A Annals of the Rheumatic Diseases 2002;61:975-980. 